\title{
Self-contamination from clothing in microplastics research
}

\section{Scopetani, Costanza}

2020-02

Scopetani , C , Esterhuizen-Londt, M , Chelazzi , D , Cincinelli , A, Setälä , H \&

Pflugmacher, S 2020 , ' Self-contamination from clothing in microplastics research ' ,

Ecotoxicology and Environmental Safety , vol. 189 , 110036 . https://doi.org/10.1016/j.ecoenv.2019.110036

http://hdl.handle.net/10138/337218

https://doi.org/10.1016/j.ecoenv.2019.110036

cc_by_nc_nd

acceptedVersion

Downloaded from Helda, University of Helsinki institutional repository.

This is an electronic reprint of the original article.

This reprint may differ from the original in pagination and typographic detail.

Please cite the original version. 


\title{
Self-contamination from clothing in microplastics research
}

\author{
Costanza Scopetani $^{\mathrm{a}, \mathrm{b}}$, Maranda Esterhuizen-Londt ${ }^{\mathrm{a}, \mathrm{d}, \mathrm{e}}$, David Chelazzi $^{\mathrm{c}}$, Alessandra Cincinelli ${ }^{\mathrm{b}, \mathrm{c}}$, \\ Heikki Setälä ${ }^{a, e}$, Stephan Pflugmacher ${ }^{a, d, e, *}$ \\ ${ }^{a}$ Faculty of Biological and Environmental Sciences Ecosystems and Environment Research Programme, University of Helsinki, Niemenkatu 73, Lahti, FI-15140, Finland \\ ' Department of Chemistry Ugo Schiff", University of Florence, 50019, Sesto Fiorentino (Florence), Italy \\ ' Department of Chemistry "Ugo Schiff”, University of Florence, And Consorzio Interuniversitario per lo Sviluppo dei Sistemi a Grande Interfase (CSGI), 50019, Sesto \\ Fiorentino (Florence), Italy \\ ${ }^{\mathrm{d}}$ Korean Institute of Science \& Technology (KIST Europe) Environmental Safety Group. Joint Laboratory of Applied Ecotoxicology, Campus E 7.1 66123, Saarbrücken, \\ Germany \\ ${ }^{\mathrm{e}}$ Helsinki Institute of Sustainability (HELSUS), Fabianinkatu 33, 00014, Helsinki, Finland
}

A R T I C L E I N F O

\section{Keywords:}

Microplastics

Self-contamination

Microplastics overestimation

\begin{abstract}
A B S T R A C T
Self-contamination should not be underestimated when quantifying microplastics (MPs) in environmental matrices. Standardised and validated methodologies for MP sampling, extraction, and analysis are lacking. The various applications of plastics in our society have made them ubiquitous, even in clothing, rendering MP selfcontamination inevitable. In the present study, we sampled lake sediment, snow, and ice, purposefully wearing red overalls composed of cotton; fibres from which we could quantify using Fourier-Transform Infrared Spectroscopy (FTIR), serving as an indication of possible self-contamination from clothes. The suitability of cotton as a representation of MP contamination was also evaluated. For all detected fibres, $25 \pm 1 \%, 20 \pm 7 \%$, and $8 \pm 6 \%$ for snow, ice, and sediment, respectively, originated from sampling attire. These findings demonstrate that self-contamination can play a significant role when quantifying MP pollution, highlighting that sampling conducted to date might have overestimated the presence of MP or even contaminated MP-free samples.
\end{abstract}

\section{Introduction}

The occurrence of microplastics (MPs), which seemingly are ubiquitous in aqueous environments (Ivar do Sul et al., 2009; Cole et al., 2011; Li et al., 2017; Barrows et al., 2018; Gago et al., 2018), has been recognised by the scientific community as well as the public as an environmental concern. Even though freshwater environments have not been investigated as much as the marine (Li et al., 2017; EerkesMedrano and Thompson, 2018; Lambert and Wagner, 2018), MP occurrence does not seem to differ between the two aqueous environments (Peng et al., 2017; Abrial et al., 2018; Scopetani et al., 2019). However, MP abundance is not homogeneously distributed within these ecosystems (Horton et al., 2017; Gago et al., 2018; Klein et al., 2018), which may result from sampling site variability, differences in collection methods, human activities (Eerkes-Medrano et al., 2015), sample treatment protocols, and analyses methods per se (Mai et al., 2018). Moreover, an increasing number of studies have detected MPs in aquatic biota at virtually all levels of the food web (Teuten et al., 2007;
Cole et al., 2011; Ugolini et al., 2013; Sanchez et al., 2014; Auta et al., 2017), bioaccumulating and posing a risk of adverse effects (Lassen et al., 2015). MPs have even been detected in remote areas such as the Arctic and Antarctic (Obbard et al., 2014; Lusher et al., 2015; Bergmann et al., 2017; Cincinelli et al., 2017; Waller et al., 2017) and analysed in beer, mineral water, and commercial sea and lake salts for human consumption (Dittmers, 2014; Karami et al., 2017). In a recent study, MPs were detected in all faecal samples from participants from eight countries (Liebmann et al., 2018). However, some of these findings have been questioned and heavily disputed, and have thus evoked the thought of possible self-contamination (Lachenmeier et al., 2015; Rist et al., 2018).

Although the number of scientific studies regarding MP pollution has increased exponentially in the last two decades, validated and standardised methods for sampling, quantification, and characterisation of MPs are still lacking. This has impeded inter-laboratory comparison, leading to reduced quality assurance and under- or overestimation of MPs (Silva et al., 2018).

\footnotetext{
* Corresponding author. Faculty of Biological and Environmental Sciences Ecosystems and Environment Research Programme, University of Helsinki, Niemenkatu 73, Lahti, FI-15140, Finland.

E-mail address: stephan.pflugmacher@helsinki.fi (S. Pflugmacher).
} 
Another issue complicating the comparison between studies is the unclear definition of MPs and limits to sizes of MP that can be detected. Hartmann et al. (2019) have highlighted how this ambiguity can lead to miscommunication and slow down the progress in research. These authors have proposed a definition for microplastics categorizing them as plastic particles in the size range of $1<$ to $1000 \mu \mathrm{m}$.

In an attempt to determine the spread of MPs in rural environments, river sediments were sampled in South America and very high amounts of MPs were detected in all of the samples (S. Pflugmacher, unpublished data). The high amounts of MPs exceeded our expectations, leading us to challenge our results and evaluate sampling protocols as critically as possible. Here we hypothesise that clothes made from synthetic materials worn during the sampling procedures represent a source of MP contamination; i.e. fibres released from the material end up in the samples mostly through airborne contamination. A quick online search on MP sampling reveals pictures of individuals wearing clothes made from synthetic fibres, thus a critical investigation of the plausibility of self-contamination was necessary. In the present study, we investigated the possibility of self-contamination resulting from sampling attire by wearing red overalls composed of cotton fibres. These fibres, which we quantified using Fourier-Transform Infrared Spectroscopy (FTIR), served as an indication of MPs self-contamination while sampling lake sediment, snow, and ice cores in the field during winter. Further, we evaluated cotton fibre release as a suitable representative for MP fibres release.

\section{Materials and methods}

\subsection{Sampling}

Sampling was conducted during the winter of 2018. To estimate the impact of self-contamination of MPs in environmental samples, three persons wearing work overalls made entirely from red natural fibres (i.e. cotton and leather) were responsible for the sampling. Sediment, snow, and ice-core samples ( $\mathrm{n}=6$ per matrix, distance between replicates 10-15 m) were collected according to Pierre Gy's theory of sampling principles (Pitard, 1993) close to the shore ( $\pm 20-30 \mathrm{~m})$ of the Pond Pikku Vesijärvi (connected to the lake Vesijärvi with a narrow channel; GPS: $60^{\circ} 59^{\prime} 19.0^{\prime \prime} \mathrm{N} 25^{\circ} 38^{\prime} 40.1^{\prime \prime} \mathrm{E}$ ) and close the shore $( \pm 100-200 \mathrm{~m})$ of the Lake Vesijärvi (GPS: $61^{\circ} 00^{\prime} 17.9^{\prime \prime} \mathrm{N}$ $\left.25^{\circ} 38^{\prime} 07.6^{\prime \prime} \mathrm{E}\right)$.

For snow and ice, $500 \mathrm{~mL}$ and $4 \mathrm{~L}$, respectively, were sampled. At that time, the lake and pond were iced over (thickness $32 \pm 4 \mathrm{~cm}$ ) completely with $6 \mathrm{~cm}$ of snow on top of the ice. The snow on top of the ice was sampled first; samples were collected from the top layer (first $5 \mathrm{~cm}$ of depth) using a metal spoon and stored in glass jars. Solid ice core samples were collected close to the places where snow was sampled with a metallic ice drill and then placed in metallic buckets covered with aluminium lids. Snow was cleared away first before drilling. Sediment (20 $\mathrm{g}$ fresh mass per replicate) was collected via a hole in the ice using the metal Ekman sampler and stored in glass jars.

Snow and ice-core samples were allowed to melt and filtered on glass fibre filters (Grade 693, $90 \mathrm{~mm}$ diameter, particle retention $1.2 \mu \mathrm{m}$, VWR) to collect fibres and particles, while fibres and fragments in the sediment samples were extracted three times through density separation according to Kovač et al. (2015) and then filtered on glass fibre filters (Grade 693, $90 \mathrm{~mm}$ diameter, particle retention $1.2 \mu \mathrm{m}$, VWR).

\subsection{Identification and analysis using FTIR}

The filters were dried and analysed using a Cary 620-670 Fourier transform infrared spectroscopy (FTIR) microscope (Agilent Technologies) equipped with an FPA (Focal Plane Array) $128 \times 128$ detector (Agilent Technologies) which allows performing 2D imagingFTIR analysis; 128 scans were acquired for each spectrum, in reflectance mode with open aperture and a spatial resolution of $4 \mathrm{~cm}-1$. Background spectra were collected directly on a gold-plated surface. The Agilent Resolution Pro software (Agilent technologies) was used to collect, process, and analyze the spectra, and to obtain 2D imaging maps. Each analysis consists in a map of $700 \mu \mathrm{m} \times 700 \mu \mathrm{m}(128 \times 128$ pixel) with an Imaging map spatial resolution of $5.5 \mu \mathrm{m}$ (i.e. each pixel has a dimension of $5.5 \mu \mathrm{m} \times 5.5 \mu \mathrm{m}$ ). Such spatial resolution allows the collection of a large number of independent spectra on fibers, for instance more than 150 independent spectra can be typically collected on a fiber of ca. $1 \mathrm{~mm}$ and $10 \mu \mathrm{m}$ thickness. By mapping the intensity of diagnostic bands in false color (red $>$ yellow $>$ green $>$ blue), it is possible to see how each band is representative of the spectra of a single fiber. A single image $(700 \mu \mathrm{m} \times 700 \mu \mathrm{m})$ was thus collected for each analysed fiber, leading to the collection of numerous independent spectra along the fiber length. The spectra showed in the FTIR images in Section 3.2 and in the Supplementary material are representative spectra from all the independent spectra for each fiber.

It is to be noted that the detection limit of an FPA detector has been found to be significantly lower than that of a conventional mercury cadmium telluride (MCT) detector for the FTIR detection of trace amounts of materials. In fact, the heterogeneous distribution of the analyte can result in small areas of localized high concentration, which can be detected due to the high spatial resolution of the FTIR FPA imaging approach (Chan and Kazarian, 2006). For instance, for polyvinyl alcohol and polyvinyl acetate we verified that quantities $<1 \mathrm{pg} /$ pixel $(1$ pixel $=5.5 \mu \mathrm{m} \times 5.5 \mu \mathrm{m})$ can be detected on reflective surfaces, i.e. smaller amounts than those we typically met in the analysis of fibers.

The 2D imaging-FTIR analysis allowed the fibres to be counted, grouped by their colour, and then characterised as cotton, plastic, or non-plastic based on their FTIR spectra. Fibres were counted and analysed in 5 randomly selected squares $2 \mathrm{~cm} \times 2 \mathrm{~cm}$ on each filter, total area $20 \mathrm{~cm}^{2}$, which comprised about $31.4 \%$ of the total filtered area. The results were normalised to the total filter surface area. All the nonaged red fibres found on the filters were analysed, and their FTIR spectra indicated they were all made of cotton (Table 1, Supplementary Information). Fibres were divided into two categories, i.e. red cotton fibres and others (composed by red synthetic fibres plus natural and synthetic fibres in all colours), in order to quantify the number of fibres coming from the red suits. Aged or bleached cotton red fibres were counted in the second category since their degradation state clearly indicated that they had been in the environment for a long time, and they did not originate from the red coveralls, nor matched the control sample cut directly from the overalls. The characterisation of cotton and MP fibres (not reported in this manuscript) was based on the assignment of the bands in Table 1, and on comparison with reference spectra in Fig. S1 (Supplementary Information).

\subsection{Cotton as a representative of MP contamination}

One old blue (4-year-old used) and one new bright pink fleece shirt as well as the three red overall were used to compare the number of fibres coming from a garment under the same conditions. First, each garment was rubbed vigorously (five different areas of the garment: neck, back, right arm, left arm, front, five times) in an enclosed white plastic bag for $5 \mathrm{~min}$. The fibres were then collected by suspension in MilliQ water, filtered on glass fibre filters (Grade 693, $90 \mathrm{~mm}$ diameter, particle retention $1.2 \mu \mathrm{m}$, VWR) before being counted. The filters were divided into $1 \mathrm{~cm} \times 1 \mathrm{~cm}$ blocks of which five blocks were counted and the total amount of fibres per garment was calculated.

\subsection{Statistical analysis}

Statistical analyses were performed in $\mathrm{R}$ (version 3.2.1) using various packages. A linear mixed model fit as applied first excluding and then including the effect of location. The differences between the fibre 


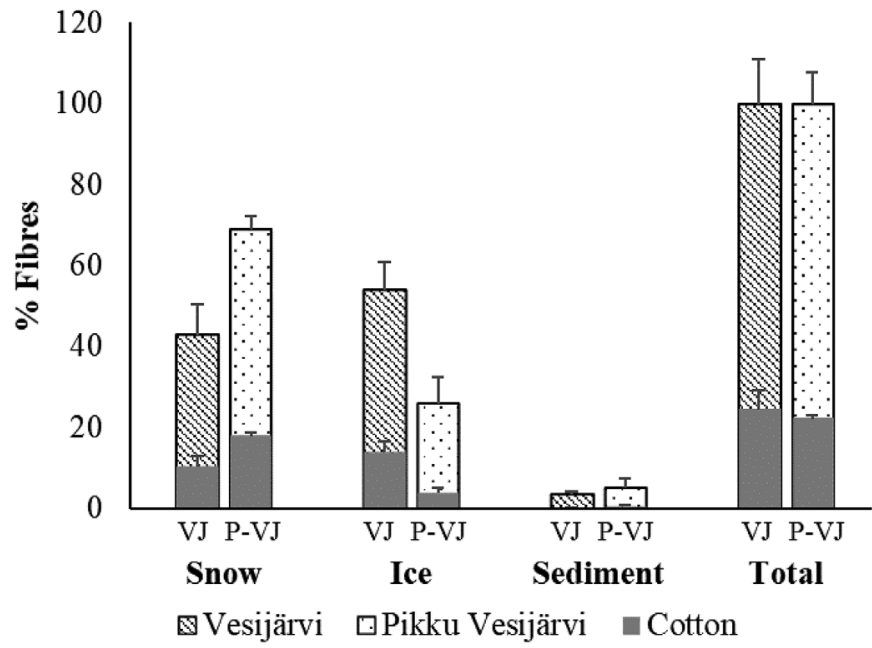

Fig. 1. Percentage distribution (mean $\pm S D ; n=6$ for each matrix type) of total fibres counted in the snow, ice, and sedimentof the Lake Vesijärvi (VJ) and Pond Pikku Vesijärvi (P-VJ) as cotton fibres (dark grey) and non-cotton fibres (stripes for Vesijärvi and dots for Pikku Vesijärvi) as well as a total count of the three matrices combined. Statistical significance is represented by asterisks $(* \mathrm{p}<0.05)$.

counts from the fleece and cotton garments were analysed by one-way analysis of variance (ANOVA) followed by Tukey's post-hoc. Significance was set to alpha of 0.05 .

\section{Results and discussion}

\subsection{Self-contamination assessment}

Fibres and fragments in the range of $100 \mathrm{~nm}$ to $5 \mathrm{~mm}$ were found in all snow, ice and sediment samples, where the fragments accounted for less than $1 \%$ of all fibres and fragments detected in total in each matrix. From all the three matrices sampled, a total average of 298 fibres was counted for both lakes combined. Non-aged (non-bleached and nonfragmented) red cotton fibres were detected in all three sample matrices from Pond Pikku Vesijärvi and Lake Vesijärvi (Fig. 1) of which on average $23 \%$ were identified as cotton using FTIR analysis (Fig. 3). The spectra of these fibres exactly matched the FTIR spectra of the fibres from the cotton overalls used during the sampling (Fig. 4). Our findings strongly suggest that self-contamination by the sampling personnel themselves can result unless effective preventive measures are taken. These data indicate that previous surveys, conducted without specific

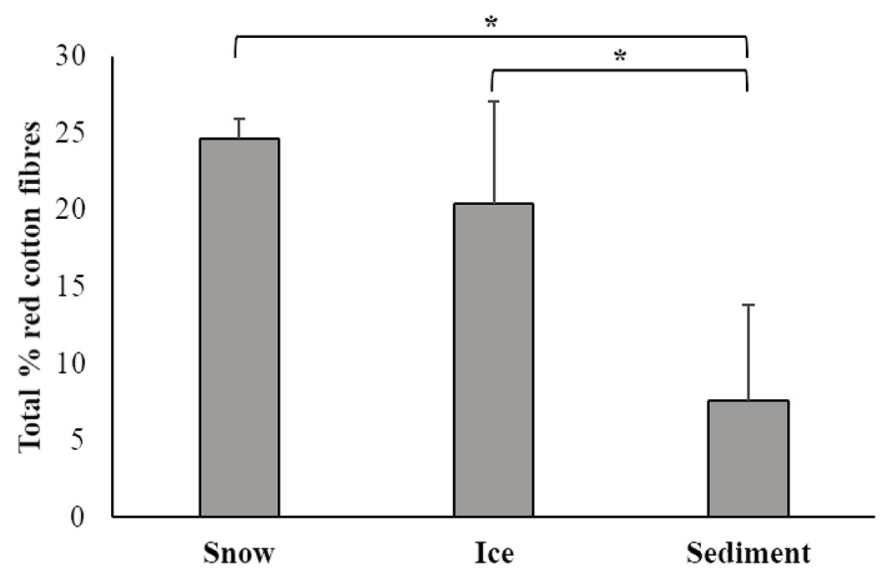

Fig. 2. Total percentage red cotton fibre of the two lakes combined. Data represent total average percentage \pm SD ( $n=6$ per matrix). Statistical significance is represented by asterisks (*p $<0.05)$.

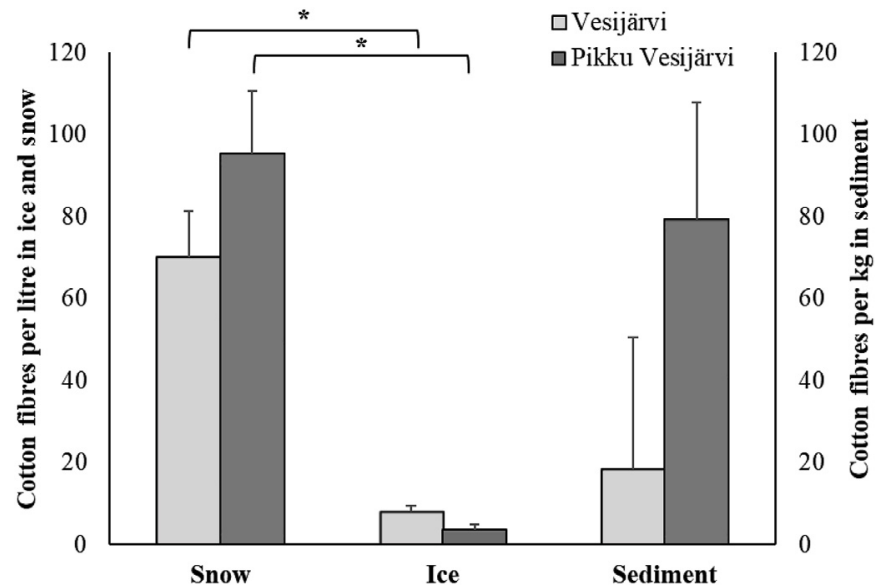

Fig. 3. Concentration of red cotton fibres in snow and ice (fibres per litre) and sediment (fibres per $\mathrm{kg}$ fresh mass). Data represent mean concentrations with standard deviation ( $n=3$ per matrix type per location). Statistical significance is represented by asterisks (*p $<0.05$ ).

sampling protocols free from contamination pitfalls, could have overestimated the presence of MPs in environmental samples.

The total percentage of red cotton fibres quantified in the three matrices sampled altogether did not differ ( $\mathrm{p}=0.55$, Fig. 1 ) between Lake Vesijärvi $(24.2 \% \pm 4.9 \%)$ and Pond Pikku Vesijärvi $(22.1 \% \pm 0.9 \%)$. However, when comparing the individual matrices, large differences were observed in the percentages which red cotton represents of the total amount of fibres. In snow, the cotton presented $10.2 \% \pm 2.7 \%$ in Lake Vesijärvi and $17.7 \% \pm 1.0 \%$ in Pond Pikku Vesijärvi, which is significantly ( $\mathrm{p}=0.03$ ) higher than that observed in the lake samples. In ice, a 3.5-times higher percentage of cotton was found in the Lake Vesijärvi samples $(13.9 \% \pm 2.6 \%)$ compared to the Pond Pikku Vesijärvi samples $(3.9 \% \pm 1.3 \%)(\mathrm{p}=0.01)$. However, in sediments, the percentages did not differ $(\mathrm{p}=0.18)$ between the lake and the pond. Despite these differences, the results indicate that selfcontamination can significantly occur regardless of the sample matrix, method, or location.

When comparing the total percentage distribution of the cotton fibres, irrespective of the sampling location (Fig. 2), the highest percentage of red fibres were found in snow, with slightly but not significantly less ( $p=0.189$ ) in ice, and the lowest in sediment $(\mathrm{p}=0.001)$. The percentage of red cotton fibres in the sediments was significantly ( $\mathrm{p}<0.001)$ lower than in snow and ice, both with and without the effect of location in the linear mixed model. Concentrations of the cotton fibres per matrix did not differ significantly $(\mathrm{p}>0.05$ ) between Lake Vesijärvi and Pond Pikku Vesijärvi (Fig. 3). The concentration of cotton fibres per litre in ice was significantly lower in snow ( $\mathrm{p}<0.001$ ). No comparison could be made to the fibre concentration in the sediment due to measurement in a different unit.

These differences in the proportion of red cotton fibres between ice, snow, and sediment samples can be attributable to the different sampling techniques used for each matrix and the amount of friction causing the release of fibres. For example, for ice core sampling, the samples were drilled and for snow the samples were shoveled which associates with a significant amount of arm movement and thus material friction, which is possibly also variable for each individual sampling, likely resulting in cotton fibres release and thus causing the higherst percentages and also the variablility (Figs. 1 and 2). The sediment samples had the lowest cotton contamination percentage and the highest standard deviation for the concentrations, likely because during the sampling they were mostly kept in the closed Ekman sampler, and thus they were less exposed to open air and therefore only some samples were contaminated.

Our results suggest that the contamination of samples by the 

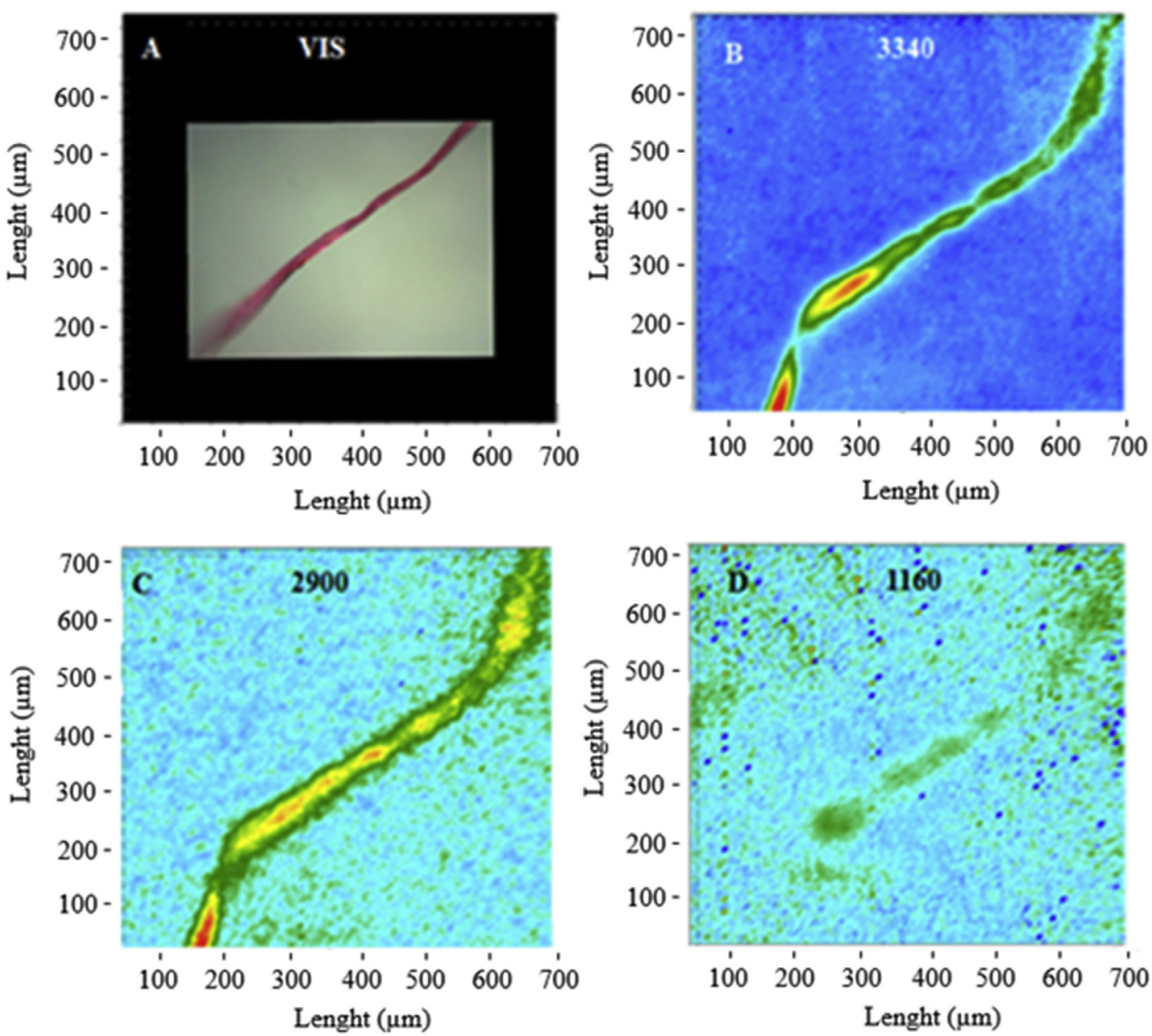

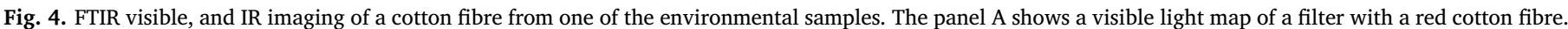

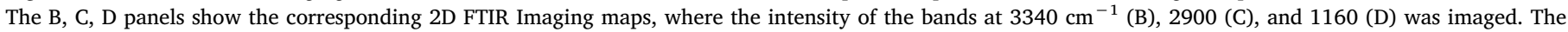

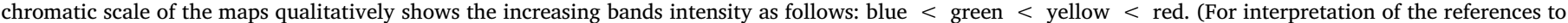
colour in this figure legend, the reader is referred to the Web version of this article.)

sampling personnel wearing inappropriate clothing can play a significant role and lead to overestimation on the occurrence of MPs in environmental samples.

\subsection{Identification and quantification of cotton}

To confirm that self-contamination can be an important source of MPs in environmental samples, FTIR analysis was performed to identify fibres and fragments smaller than $5 \mathrm{~mm}$ up to few tens of microns in the ice, snow, and sediment samples. Cotton was identified and distinguished from "other" fibres and FTIR data confirmed that the nonaged red fibres found in our samples were cotton.

Fig. 3 shows part of a red cotton fibre of ca. $800 \mu \mathrm{m}$ in length. The spectra show intense bands, clearly distinguishable from the filter's absorptions at $3340 \mathrm{~cm}^{-1}$ (stretching OH; see Methods), $2900 \mathrm{~cm}^{-1}$ (stretching $\mathrm{CH}$ ), $1635 \mathrm{~cm}^{-1}$ (adsorbed water), $1160 \mathrm{~cm}^{-1}$ (stretching $\mathrm{C}-\mathrm{C}$ ring breathing), and $1025 \mathrm{~cm}^{-1}$ (stretching $\mathrm{C}-\mathrm{OH}$ ) which are characteristics of cellulose (Garside and Wyeth, 2003). The intensity of the peaks at $3340 \mathrm{~cm}^{-1}, 2900 \mathrm{~cm}^{-1}$, and $1160 \mathrm{~cm}^{-1}$ was imaged, which gave an IR map in agreement with the fibre profile in the visible image. The assignment was confirmed by Attenuated Total Reflectance (ATR) FTIR analysis, namely the ATR spectra of the fiber was a perfect match with the reference spectra of cotton reported by Garside et al. (Garside and Wyeth, 2003).

These findings strongly suggest that the red fibres detected in the environmental samples derived from the overall worn by the sampling personnel.

To further confirm whether the cotton indeed originated from the sampling overalls, samples were taken directly from the red overall, and the FTIR ATR and Reflectance spectra were compared to that of the non-aged red fibres found in the environmental samples. Fig. 4 shows part of a fibre directly collected from the red overalls. The FTIR ATR and Reflectance spectra were acquired, and both spectra exhibit characteristic bands at $3364 \mathrm{~cm}^{-1}$ (stretching OH), $2910 \mathrm{~cm}^{-1}$ (stretching $\mathrm{CH}), 1639 \mathrm{~cm}^{-1}$ (adsorbed water), $1153 \mathrm{~cm}^{-1}$ (stretching C-C ring breathing), and $1025 \mathrm{~cm}^{-1}$ (stretching $\mathrm{C}-\mathrm{OH}$ ) which are characteristics of cellulose (see Table 1, Supplementary Information), and perfectly matched the reference spectrum of cotton (Garside and Wyeth, 2003). The intensity of the peaks at 3364,2910 , and $1639 \mathrm{~cm}^{-1}$ was imaged, which gave IR maps in good agreement with the fibre profile in the visible image. The two ATR spectra reported in Figs. 3 and 4 match perfectly and thus it can be expected that the fibre represented in Fig. 3 originated from the red overalls worn during sampling.

This analysis supports the idea of possible contamination of samples by sampling personnel and highlights the importance of performing FTIR analysis in order to distinguish synthetic polymers from other materials. Indeed, besides from red cotton fibres also plastic items were found in snow, ice and sediment samples. The composition of all fibres and fragments in the range of $100 \mathrm{~nm}$ to $5 \mathrm{~mm}$ detected in the three matrices were analysed, including MP (identification criteria in Table 1, Supplementary Information in order to differentiate between cotton, MP, and other materials with certainty. Fig. 5 shows an example of a 

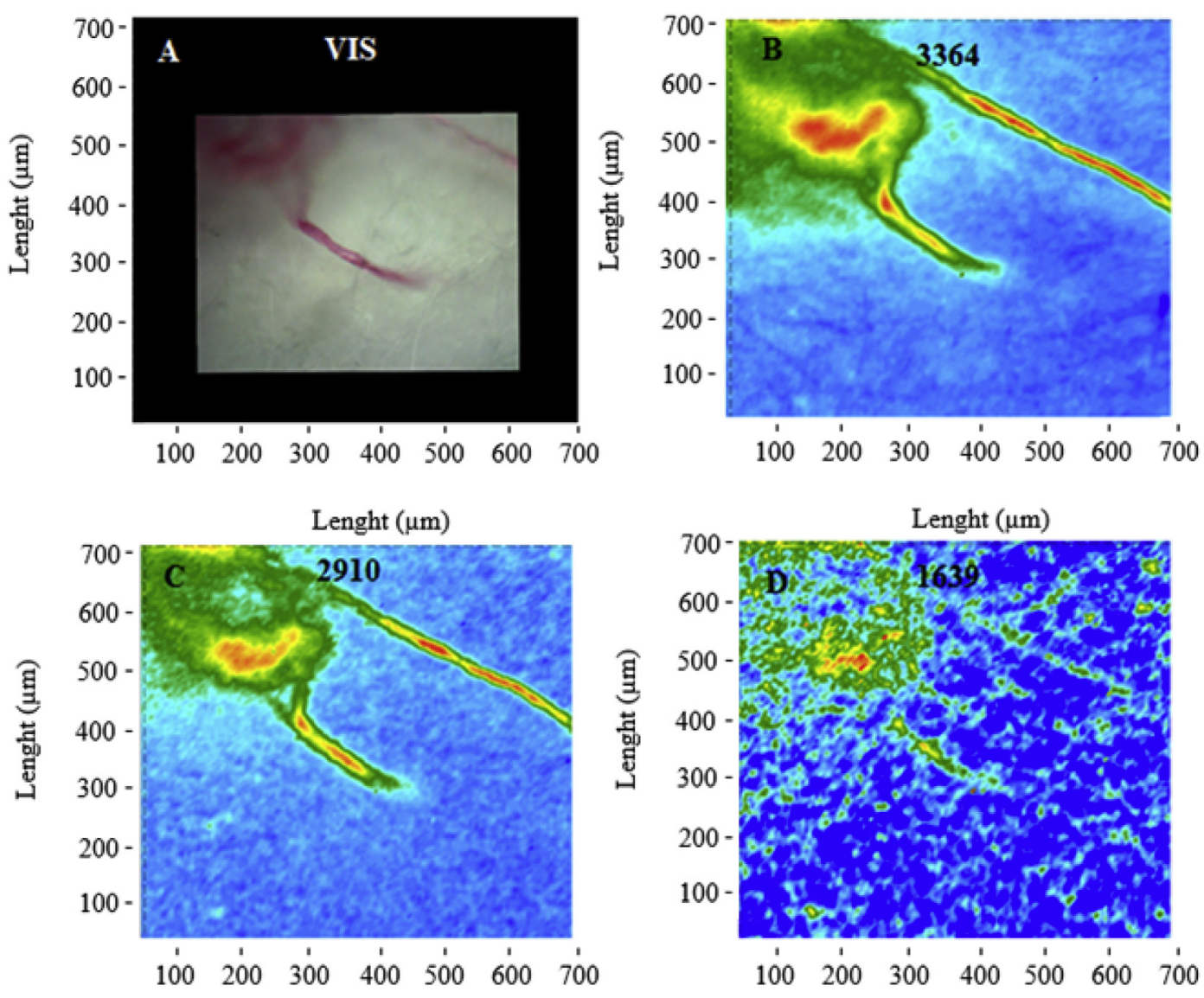

Lenght $(\mu \mathrm{m})$

Lenght $(\mu \mathrm{m})$

Fig. 5. FTIR visible, and IR imaging of an overall fibre. The panel A shows a visible light map of a red cotton fibre, taken directly from the overall. The B, C, D panels show the corresponding 2D FTIR Imaging maps, where the intensity of the bands at $3364 \mathrm{~cm}^{-1}$ (B), 2910 (C), and 1639 (D) was imaged. The chromatic scale of the maps qualitatively shows the increasing intensity of the bands as follows: blue $<$ green $<$ yellow $<$ red. (For interpretation of the references to colour in this figure legend, the reader is referred to the Web version of this article.)

fibre detected in an ice sample identified as polypropylene due to intense absorption peaks between 3000 and $2800 \mathrm{~cm}^{-1}$ (CH stretching region), at $1458\left(\delta \mathrm{CH}_{2}\right), 1377\left(\delta \mathrm{CH}_{3}\right)$ and $1165 \mathrm{~cm}^{-1}$ ( $\mathrm{CH}$ bend, $\mathrm{CH}_{3}$ rock, C-C stretching) (Andreassen, 1999; Verleye et al., 2001; Noda et al., 2007; Asensio et al., 2009; Jung et al., 2018). In fact, the wavenumber, relative intensity and shape of the peaks match with those of the reference spectrum of polypropylene (see Fig. S1, Supplementary Information). The intensities of the peaks at 2916, 1458, 1377 and $1165 \mathrm{~cm}^{-1}$ were imaged, providing IR maps in good agreement with the fibre profile in the visible image (Table 1, Supplementary Information) (see Fig. 6).

\subsection{Cotton vs MPs release}

In order to understand whether synthetic clothing worn during the sampling could be a real source of MPs contamination and to evaluate the impact of this self-contamination, the red overalls worn during sampling and one 4-year-old and one new fleece jacket were used in a rubbing experiment to directly compare the fibre release in a set amount of time. This experiment allowed to test whether the release of cotton fibres from a cotton overall adequately resembles the release of MP fibres from a synthetic material. We realized that cotton could be more susceptible for shedding fibers and this is the reason why we performed the cotton vs. MPs release experiment. The results show that cotton tends to release more fibres than the fleece jackets. The same ( $p=0.999$ ) amount of fibres were released from the 4-year-old versus the new fleece jacket (Fig. 7). The release of cotton fibres from the overalls was, however, 36\% ( $\mathrm{p}<0.001)$ higher than the average amount of MP fibres released from the fleece jackets. This trend was consistent even after five repetitions of rubbings from five different parts of the garments, and thus cotton still represented a reproducible indication of self-contamination to assess possible MP fibre release and thus self-contamination during environmental sampling.

Our data show that MP self-contamination can have an uncontrolled overestimating effect on MP concentrations in environmental samples, manifesting the importance of avoiding self-contamination in future MPs research. An average of $23 \%$ of the fibres detected in our environmental samples was from self-contamination, suggesting that up to $15 \%$ (normalised for the release difference between MP and cotton) of MPs in environmental samples could be a result of self-contamination. We showed that the impact of self-contamination can vary depending on the sample matrix, how long the samples are exposed to air, and the tendency of the clothes to release fibres. It should also be noted that self-contamination could occur not only during sampling but also during treatment and analysis of samples.

We strongly recommend avoiding any synthetic clothing during all the steps of the working process. When the climate conditions of the sampling sites are prohibitive and warm technical synthetic fabrics are needed to be worn, we suggest to wear cotton jumpsuit over them (like we did in this study).

Furthermore, weather conditions like rain and the wind speed and direction could also affect the impact of self-contamination during the sampling. All these variables have to be taken into consideration when coping with data correction and further studies are needed to determine 

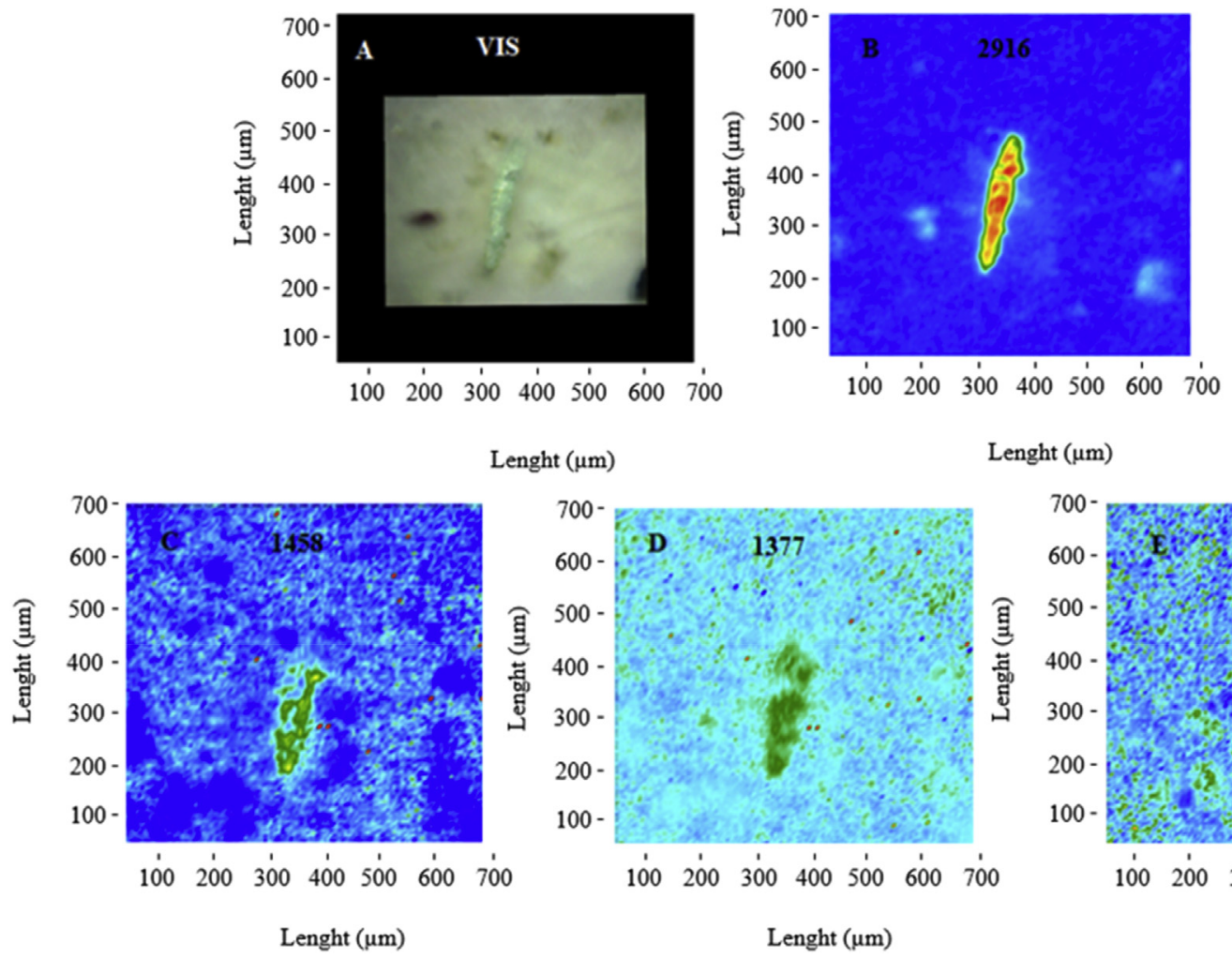

Lenght $(\mu \mathrm{m})$

Lenght $(\mu \mathrm{m})$
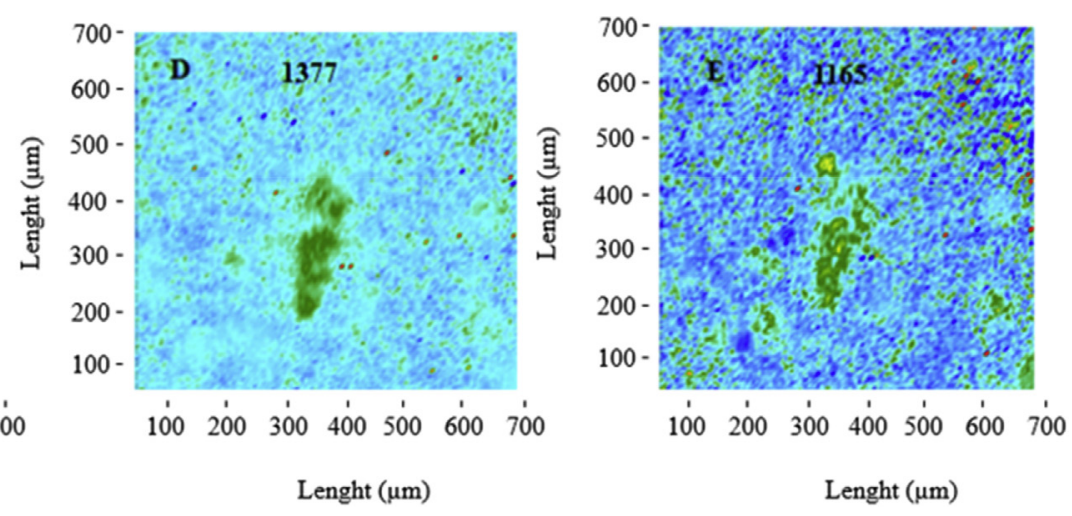

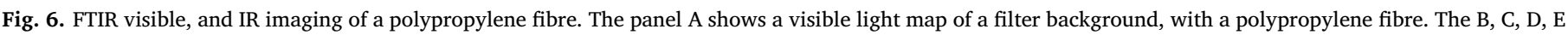

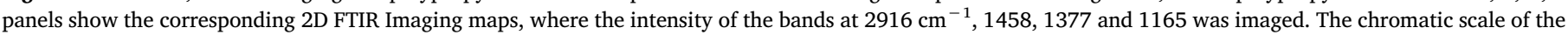

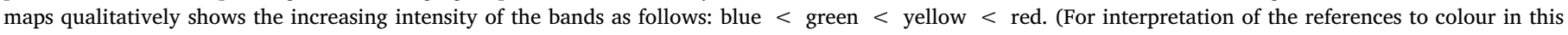
figure legend, the reader is referred to the Web version of this article.)

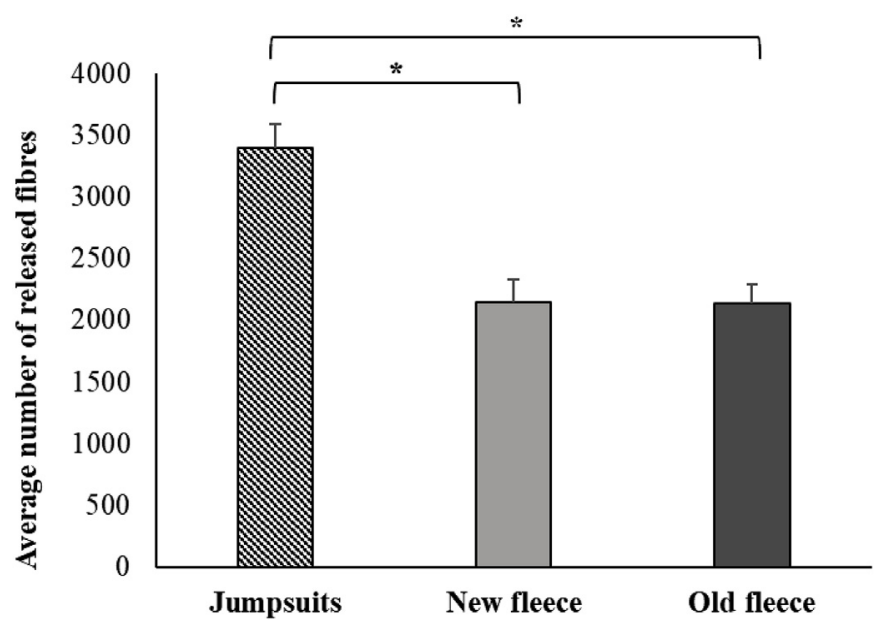

Fig. 7. Fibres released from an old and a new fleece jacket compared to the cotton overalls. Data represent the average number of fibres released per garment repeated five times at five locations (neck, back, left arm, right arm, front) \pm standard deviation $(n=5)$. Statistical significance is represented by asterisks (*p $<0.05)$.

the impact of each of these factors.

The technical memorandum published by NOAA (Masura et al., 2015) suggests a protocol to quantify MPs in marine waters and sediments. Our results suggest that such a protocol would be insufficient unless it includes self-contamination precautions. The technical BASEMAN report (2018) identifies some useful procedure to reduce cross contamination risks, and recently the GESAMP protocol (2019) has underlined how the problem of self-contamination has not received adequate attention so far, questioning the reliability of some published studies. Also, the legislative framework is hampered by the lack of validated analytical methods.

\section{Conclusions}

Our work on the three environmental matrices provides clear evidence that contamination from fibres stemming from sources other than the targeted samples should not be underestimated. These results indicate that research studies planned without paying attention in avoiding possible contamination sources such as synthetic cloths, might overestimate the occurrence of MPs. Consequently, standardised and validated protocols free from self-contamination should be demanded to provide reliable data to set MP thresholds for regulatory guidelines, and to allow policy-makers to focus on the problems that microplastics may bring about.

\section{Authors contributions section}

Costanza Scopetani: Conventionalization and experimental design, Sampling, Analysis, Manuscript preparation. Maranda EsterhuizenLondt: Conventionalization and experimental design, Sampling, Analysis, Manuscript preparation. David Chelazzi: Manuscript preparation. Alessandra Cincinelli: Manuscript preparation. Heikki Setälä: Conventionalization and experimental design, Manuscript preparation. Stephan Pflugmacher: Conventionalization and experimental design, Sampling, Manuscript preparation.

\section{Declaration of competing interest}

None. 


\section{Acknowledgements}

We thank Tuukka Ryynänen and Changyi Lu for assisting with sampling, Hannah Madler and Victoriia Semenova for valuable help in the laboratory, and Johan Kotze for statistical assistance. Financial support from the University of Helsinki within the start-up funds for the Professorship of Aquatic Ecotoxicology in an Urban Environment. The Consorzio Interuniversitario per lo Sviluppo dei Sistemi a Grande Interfase (CSGI), Florence, is also gratefully acknowledged.

\section{Appendix A. Supplementary data}

Supplementary data to this article can be found online at https:// doi.org/10.1016/j.ecoenv.2019.110036.

\section{Data availability}

The data that support the findings of this study are available from the corresponding author upon request.

\section{References}

Abrial, E., Khan, F.R., Espinola, L.A., 2018. Freshwater plastic pollution: recognizing research biases and identifying knowledge gaps. Water Res. 143, 416-424.

Andreassen, E., 1999. Infrared and Raman spectroscopy of polypropylene. In: KargerKocsos, J. (Ed.), Polypropylene: an A-Z Reference. Springer, Dordrecht, pp. 320-328 Polymer Science and Technology Series.

Asensio, R.C., Moya, M.S.A., de la Roja, J.M., Gómez, M., 2009. Analytical characterization of polymers used in conservation and restoration by ATR-FTIR spectroscopy. Anal. Bioanal. Chem. 395 (7), 2081-2096.

Auta, H.S., Emenike, C.U., Fauziah, S.H., 2017. Distribution and importance of microplastics in the marine environment: a review of the sources, fate, effects, and potential solutions. Environ. Int. 102, 165-176.

Barrows, A.P.W., Cathey, S.E., Petersen, C.W., 2018. Marine environment microfiber contamination: global patterns and the diversity of microparticle origins. Environ. Pollut. 237, 275-284.

Bergmann, M., et al., 2017. High quantities of microplastic in Arctic deep-sea sediments from the HAUSGARTEN observatory. Environ. Sci. Technol. 51, 11000-11010.

Chan, K.L.A., Kazarian, S.G., 2006. Detection of trace materials with Fourier transform infrared spectroscopy using a multi-channel detector. Analyst 131, 126-131.

Cincinelli, A., et al., 2017. Microplastic in the surface waters of the Ross Sea (Antarctica): occurrence, distribution and characterization by FTIR. Chemosphere 175, 391-400.

Cole, M., Lindeque, P., Halsband, C., Galloway, T.S., 2011. Microplastics as contaminants in the marine environment: a review. Mar. Pollut. Bull. 62, 2588-2597.

Dittmers, H., 2014. Mikroplastik in Mineralwasser und Bier. https://www.ndr.de/ ratgeber/verbraucher/Mikroplastik- in- Mineralwasser- und- Bier,mikroplastik134. html.

Eerkes-Medrano, D., Thompson, R.C., Aldridge, D.C., 2015. Microplastics in freshwater systems: a review of the emerging threats, identification of knowledge gaps and prioritisation of research needs. Water Res. 75, 63-82.

Eerkes-Medrano, D., Thompson, R.C., 2018. Chapter 4 - Occurrence, Fate, and Effect of Microplastics in Freshwater Systems. Microplastic Contamination in Aquatic Environments. Elsevier, pp. 95-132.

Gago, J., Carretero, O., Filgueiras, A.V., Viñas, L., 2018. Synthetic microfibers in the marine environment: a review on their occurrence in seawater and sediments. Mar. Pollut. Bull. 127, 365-376.

Garside, P., Wyeth, P., 2003. Identification of cellulosic fibres by FTIR spectroscopy: thread and single fibre analysis by attenuated total reflectance. Stud. Conserv. 48, 269-275.

GESAMP, 2019. Guidelines or the monitoring and assessment of plastic litter and microplastics in the ocean. In: Kershaw, P.J., Turra, A., Galgani, F. (Eds.), (IMO/FAO/ UNESCO-IOC/UNIDO/WMO/IAEA/UN/UNEP/UNDP/ISA Joint Group of Experts on the Scientific Aspects of Marine Environmental Protection). Rep. Stud. GESAMP No. vol. 99. pp. 130p.

Hartmann, N.B., Hüffer, T., Thompson, R.C., Hassellöv, M., Verschoor, A., Daugaard, A.E., Rist, S., Karlsson, T., Brennholt, N., Cole, M., Herrling, M.P., Hess, M.C., Ivleva, N.P., Lusher, A.L., Wagner, M., 2019. Are we speaking the same language? Recommendations for a definition and categorization framework for plastic debris.
Environ. Sci. Technol. 53, 1039-1047.

Horton, A.A., Walton, A., Spurgeon, D.J., Lahive, E., Svendsen, C., 2017. Microplastics in freshwater and terrestrial environments: evaluating the current understanding to identify the knowledge gaps and future research priorities. Sci. Total Environ. 586, $127-141$.

Ivar do Sul, J.A., Spengler, A., Costa, M.F., 2009. Here, there and everywhere. Small plastic fragments and pellets on beaches of Fernando de Noronha (Equatorial Western Atlantic). Mar. Pollut. Bull. 58 (8), 1236-1238.

Jung, M.R., et al., 2018. Validation of ATR FT-IR to identify polymers of plastic marine debris, including those ingested by marine organisms. Mar. Pollut. Bull. 127, 704-716.

Karami, A., et al., 2017. The presence of microplastics in commercial salts from different countries. Sci. Rep. 7, 46173.

Klein, S., Dimzon, I.K., Eubeler, J., Knepper, T.P., 2018. Analysis, Occurrence, and Degradation of Microplastics in the Aqueous Environment. Freshwater Microplastics. Springer Open, Chen, pp. 51-67.

Kovač, V.M., Palatinus, A., Kaberi, H., Tsangaris, C., Mazziotti, C., 2015. Recommendation on Regional Approach to Monitoring and Assessment of Microplastic in the Marine Environment. The Document Produced within the Project DeFishGear ( $\left.1^{\circ} \mathrm{str} / 00010\right)$. IPA Adriatic Cross-border Cooperation Programme 2007-2013.

Lachenmeier, D.W., Kocareva, J., Noack, D., Kuballa, T., 2015. Microplastic identification in German beer - an artefact of laboratory contamination? Dtsch. Lebensm.-Rundsch. $111,4$.

Lambert, S., Wagner, M., 2018. Microplastics Are Contaminants of Emerging Concern in Freshwater Environment: an Overview. Freshwater Microplastics, the Handbookof Environmental Chemistry. Springer Open, Cham, pp. 1-23.

Lassen, C., et al., 2015. Microplastics: Occurrence, Effects and Sources of Releases to the Environment in Denmark. Danish Environmental Protection Agency, Copenhagen.

Li, J., Liu, H., Chen, J.P., 2017. Microplastics in freshwater systems: a review on occurrence, environmental effects, and methods for microplastics detection. Water Res. 137, 362-374.

Liebmann, B., et al., 2018. Assessment of microplastic concentrations in human stool preliminary results of a prospective study. In: Conference: Int. Conference on Emerging Contaminants (EMCON).

Lusher, A.L., Tirelli, V., O'Connor, I., Officer, R., 2015. Microplastics in Arctic polar waters: the first reported values of particles in surface and sub-surface samples. Sci. Rep. 5, 14947.

Mai, L., Bao, L.-J., Shi, L., Wong, C.S., Zeng, E.Y., 2018. A review of methods for measuring microplastics in aquatic environments. Environ. Sci. Pollut. Res. 25, 11319-11332.

Masura, J., Baker, J., Foster, G., Arthur, C., 2015. Laboratory methods for the analysis of microplastics in the marine environment: recommendations for quantifying synthetic particles in waters and sediments. NOAA Marine Debris Program 1-31.

Noda, I., Dowrey, A.E., Haynes, J.L., Marcott, C., 2007. Group frequency assignments for major infrared bands observed in common synthetic polymers. In: Mark, J.E. (Ed.), Physical Properties of Polymers Handbook. Springer Science + Business Media, LLC, New York, pp. 395-406.

Obbard, R.W., et al., 2014. Global warming releases microplastic legacy frozen in Arctic Sea ice. Earth's Future 2 (6), 315-320.

Peng, J., Wang, J., Cai, L., 2017. Current understanding of microplastics in the environment: occurrence, fate, risks, and what we should do. Integr. Environ. Assess. Manag. 13 (3), 476-482.

Pitard, F.F., 1993. Pierre Gy's Sampling Theory and Sampling Practice. CRC Press, Boca Raton, Florida.

Rist, S., Almroth, B.C., Hartmann, N.B., Karlsson, T.M., 2018. A critical perspective on early communications concerning human health aspects of microplastics. Sci. Total Environ. 626, 720-726.

Sanchez, W., Bender, C., Porcher, J.M., 2014. Wild gudgeons (Gobio gobio) from French rivers are contaminated by microplastics: preliminary study and first evidence. Environ. Res. 128, 98-100.

Scopetani, C., Chelazzi, D., Cincinelli, A., Esterhuizen-Londt, M., 2019. Assessment of microplastic pollution: occurrence and characterization in Vesijärvi lake and Pikku Vesijärvi pond, Finland. Environ. Monit. Assess. 191 (11), 1-17 17 pp., 652.

Silva, A.B., et al., 2018. Microplastics in the environment: challenges in analytical chemistry-A review. Anal. Chim. Acta 1017, 1-19.

Teuten, E.L., Rowland, S.J., Galloway, T.S., Thompson, R.C., 2007. Potential for plastic to transport hydrophobic contaminants. Environ. Sci. Technol. 41, 7759-7764.

Ugolini, A., Ungherese, G., Ciofini, M., Lapucci, A., Camaiti, M., 2013. Microplastic debris in sandhoppers. Estuar. Coast Shelf Sci. 129, 19-22.

Verleye, G.A., Roeges, N.P., De Moor, M.O., 2001. Easy Identification of Plastics and Rubbers. Rapra Technology Limited, Shropshire, pp. 174.

Waller, C.L., et al., 2017. Microplastics in the Antarctic marine system: an emerging area of research. Sci. Total Environ. 598, 220-227. 